\title{
Neutravidin biosensor for direct capture of dual-functional biotin-molecular beacon-AuNP probe for sensitive voltammetric detection of microRNA
}

Zina Fredj, Sawsen Azzouzi, Anthony Turner, Ben Ali, Mounir and Wing Cheung Mak

Journal Article

\section{Tweet}

N.B.: When citing this work, cite the original article.

Original Publication:

Zina Fredj, Sawsen Azzouzi, Anthony Turner, Ben Ali, Mounir and Wing Cheung Mak, Neutravidin biosensor for direct capture of dual-functional biotin-molecular beacon-AuNP probe for sensitive voltammetric detection of microRNA, Sensors and actuators. B, Chemical, 2017. 248(), pp.77-84.

http://dx.doi.org/10.1016/j.snb.2017.03.160

Copyright: Elsevier

http://www.elsevier.com/

Postprint available at: Linköping University Electronic Press

http://urn.kb.se/resolve?urn=urn:nbn:se:liu:diva-137576

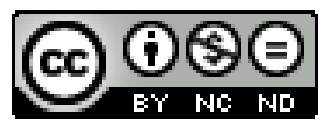


Neutravidin biosensor for direct capture of dual-functional biotin-molecular beacon-AuNP probe for sensitive voltammetric detection of microRNA

(1) Zina Fredja,b,\#, Sawsen Azzouzi ${ }^{\mathrm{a}, \mathrm{b}, \#}$, Anthony P.F. Turner ${ }^{\mathrm{a}}$, Mounir Ben Ali ${ }^{\mathrm{b}}$, Wing Cheung Mak ${ }^{\mathrm{a}, *}$

${ }^{a}$ Biosensors and Bioelectronics Centre, Department of Physics, Chemistry and Biology (IFM), Linköping University, S-58183 Linköping, Sweden

${ }^{b}$ University of Sousse, Higher Institute of Applied Sciences and Technology of Sousse, GREENS-ISSAT, Cité Ettafala, 4003 Ibn Khaldoun Sousse, Tunisia

\section{${ }^{\#}$ These authors contributed equally with joined first authorship}

\section{${ }^{*}$ Corresponding authors:}

e-mail: wing.cheung.mak@liu.se

\section{Abstract:}

We have demonstrated a new approach using a neutravidin-based biosensor combined with a dual-function gold nanoparticle (AuNP) biolabel, for simple and sensitive detection of microRNA-21 (miRNA-21). The selectivity of the biosensor is provided by the intrinsic properties of the dual-functional biotin-MB-AuNP label. The assay procedure is relatively simple, exploiting a one-pot assay concept where the affinity capture of the miRNA-21/dualfunctional biotin-MB-AuNP complex, via the strong biotin-neutravidin supramolecular interaction, and simultaneous detection of the captured AuNPs label with stripping voltammetry, is performed in a single step. This electrochemical miRNA biosensor could detect miRNA-21 with limit of detection of $0.1 \times 10^{-12}$ and a dynamic range from $0.5 \times 10^{-12}$ to $1.0 \times 10^{-}$ ${ }^{9} \mathrm{M}$. The performance of the miRNA-21biosensor was further improved after silver deposition onto the AuNPs, delivering an enhanced detection limit of $4.0 \times 10^{-15} \mathrm{M}$ of miRNA-21, and an extremely wide analytic dynamic range from $10 \times 10^{-15}$ to $1 \times 10^{-9} \mathrm{M}$ (5 orders of magnitude). This exceptionally broad dynamic range demonstrates the advantage of the one-pot assay approach with direct capture of the dual functional biotin-MB-AuNP via the strong biotin- 
neutravidin supramolecular interaction. Furthermore, we demonstrated the detection of miRNA-21 in spiked serum at clinically relevant concentrations. The miRNA biosensor displayed excellent analytical performance for the detection of miRNA and could provide a powerful and convenient tool for biomedical research and applications in cancer diagnostics.

Keywords: neutravidin; biosensor; microRNA; molecular beacon; stripping voltammetry

\section{Introduction:}

MicroRNAs (miRNAs) are short (18-22 nucleotides) non-coding RNA sequences. They were first identified in nematodes, in 1993, by Lee et al. [1] and to date, over 1000 separate miRNA sequences have been identified in the human [2]. It is becoming clear that miRNAs represent a vast, previously unrecognised level of molecular signaling in eukaryotes, and that miRNAs play an important role in the regulation of protein expression [3] and a significant role in several biological processes including: cell proliferation, developmental regulation, differentiation and epigenetic inheritance [4]. Recent studies have shown that the levels of miRNAs in body fluid can be correlated to the cancer type [5], especially, in prostate cancer (PCa), which is the second most common malignancy and the fifth leading cause of cancer death in men worldwide [6]. In most European countries such as France, The Netherlands, and the Czech Republic, the PCa incidence increased significantly in the early 1990s, and is still increasing $[7,8]$.

Current standard methods for identification and quantification of miRNAs are based on traditional molecular biology techniques (Northern blot, microarray, qRT-PCR). Although these approaches are very sensitive and reliable, they are often expensive, time consuming and need highly trained technicians $[9,10]$. Hence, there is a real challenge to develop devices able to simultaneously detect and easily quantify different miRNA sequences. Electrochemical biosensors offer the advantage of being amenable to mass fabrication at low cost and hence facilitate decentralised analysis [11].

Various electrochemical methods are available for the determination of miRNA. Gao et. al. reported an amperometric assay for the measurement of miRNAs with a detection limit of 80 $\times 10^{15} \mathrm{M}$, using an oligonucleotide capture probe immobilised onto an indium tin oxide (ITO) electrode [12]. In the same context, Peng et al. developed an amperometric miRNA biosensor, which delivered a sensitive analysis of miRNA with a detection limit of $2.0 \times 10^{-15} \mathrm{M}$ [13]. Yin et al. described a biosensor which exhibited excellent sensitivity and a low detection limit of $60 \times 10^{-15} \mathrm{M}$, based on dendritic gold nanostructures and a graphene nanosheet-modified glassy 
carbon electrode [14]. Bettazzi et al. developed an amperometric biosensor for miRNA detection based on para-magnetic beads and enzyme amplification [15]. Peng et al. reported an impedimetric miRNA biosensor based on the combination of $\mathrm{RuO} 2$ nanoparticles and the catalytic deposition of poly (3,3'-dimethoxybenzidine) (PDB) [16]. Kilic and his colleagues reported a highly sensitive voltammetric assay for detection of miRNA based on the immobilization of the oligonucleotide capture probes onto a pencil graphite electrode (PGE) [17]. A voltammetric biosensor based on oligonucleotide encapsulated silver nanoclusters (AgNCs) that could detect as low as $67 \times 10^{-15} \mathrm{M}$ of miRNA was reported by Dong and coworkers [18]. Bartosik and colleagues developed a miRNA assay by immobilising a biotin-labeled oligonucleotide capture probe onto the surface of streptavidin-coated magnetic beads [19]. Zhou and coworkers developed a miRNA biosensor based on mimicking enzyme catalysis and signal amplification [20]. In this work, gold nanoparticles were electrochemically deposited onto the surface of a glassy carbon electrode. Recently, Liu et al. developed a voltammetric biosensor detecting miRNA levels down to $3.0 \times 10^{-15} \mathrm{M}$ in which the target miRNA was hybridised with a pre-immobilised DNA capture probe onto the surface of a gold electrode [21]. Nevertheless, the above electrochemical methods are based on relatively complex and tedious sensor surface preparation with immobilised oligonucleotide capture probe, and require multiple hybridisation steps.

In the present work, we report the development of a robust neutravidin based biosensor for voltammetric detection of miRNA. The assay selectivity was provided by the smart design of a dual-functional biotin-MB-AuNP probe with a one-pot assay concept, such that the affinity capture of the miRNA-21/dual-functional biotin-MB-AuNP complex occurred via the strong biotin-neutravidin supramolecular interaction, and stimulations detection of the captured AuNPs label was achieved within a single step. The performance of the voltammetric miRNA21 biosensor was greatly improved after silver deposition onto the AuNPs, which allowed the detection of miRNA-21 with a broad analytic dynamic range from $10 \times 10^{-15}$ to $1.0 \times 10^{-6} \mathrm{M}$. The fabrication and binding processes of the miRNA biosensor were characterised with stripping square wave voltammetry.

\section{Materials and Methods}

\subsection{Materials}

Sulfuric Acid $\left(\mathrm{H}_{2} \mathrm{SO}_{4}\right)$, nitric acid $\left(\mathrm{HNO}_{3}\right)$, sodium hydroxide, sodium chloride, sodium citrate, Neutravidin and silver enhancement kit were purchased from Sigma Aldrich (St. Louis, MO, USA). All chemicals used in this study were of analytical reagent grade. All solutions 
were prepared with ultrapure (18.2 M 2 ) water from a Millipore Milli-Q water purification system (Billerica, MA).The sequence of the DNA/LNA MBs was taken from previous reports[22]. The presence of a thiol group at the 3' end of the MB allowed its immobilisation onto the AuNPs, while at the other end the biotin at the 5' was use to facilitate the capture of the biotin-MB-AuNP/miRNA complex onto the transducer surface via interaction with an immobilised Neutravidin layer.

To facilitate the handling of the sample, RNA mimic sequences were taken from miRBase (http://www.mirbase.org) and synthesised by biomers.net (Germany). LNA modified Oligonucleotide probes were obtained from Exiqon (Denmark):

$M B:$

\section{5'-/5BioTEG/GGCCGTCAACATCAGTCTGATAAGCTACGGCCTTTTTTTTTT/} 3ThioMC3-D/-3' (in bold and italics are the LNA bases) miRNA-21:5'-UAGCUUAUCAGACUGAUGUUGA-3' miRNA-205: 5'-UCCUUCAUUCCACCGGAGUCUGU-3' miRNA-221: 5'-AGCUACAUUGUCUGCUGGGUUUC-3'

Oligonucleotide stock solutions $(100 \mu \mathrm{M})$ were prepared by dissolving the lyophilised synthetic sequences in filtered (filter size: $0.2 \mu \mathrm{m}$ ) MilliQ water. All stock solutions were stored at $-20^{\circ} \mathrm{C}$. To reduce the risks of deactivation of the thiol group, the stock solution of the MB was divided in aliquots that were stored at $-20{ }^{\circ} \mathrm{C}$ and defrosted only when needed.

\subsection{Instrumentation}

Stripping square wave voltammetry (SSWV) was performed using an IviumStat Potentiostat/Galvanostat (Ivium, The Netherlands) with a three-electrode cell. A glassy carbon (GC) electrode ( $2 \mathrm{~mm}$ in diameter, CHI Instruments) was used as the working electrode. An $\mathrm{Ag} / \mathrm{AgCl} \mathrm{KCl} 3 \mathrm{M}$ (CHI Instruments) electrode and a platinum wire were used as the reference and counter electrodes, respectively. All the potential values presented are vs. a $\mathrm{Ag} / \mathrm{AgCl} \mathrm{KCl}$ $3 \mathrm{M}$ reference. The voltammetry measurements were performed in $0.1 \mathrm{M}$ sulphuric acid buffer solution before silver enhancement and $10 \mathrm{mM}$ nitric acid as buffer solution after silver enhancement. The amplitude of the applied sine wave potential was $5 \mathrm{mV}$.

A Zetasizer Nano ZS90 (Malvern Instruments Ltd., Worcestershire, UK) using dynamic light scattering was used to measure the size and zeta potentials of the AuNPs and biotin-MBAuNPs conjugate. The mean size and zeta potential values were calculated by taking an average of 3 repeated measurements and were performed at room temperature $\left(20^{\circ} \mathrm{C}\right)$. 


\subsection{Preparation of gold nanoparticles (AuNPs)}

AuNPs were prepared according to the literature [22] by the citrate reduction of $\mathrm{HAuCl}_{4}$. In brief, $50 \mathrm{~mL}$ of $1 \mathrm{mM} \mathrm{HAuCl}_{4}$ were brought to boil under vigorous stirring. Rapid addition of $5 \mathrm{~mL}$ of a $38.8 \mathrm{mM}$ sodium citrate solution to the vortex of the solution resulted in a colour change from pale yellow to burgundy. Boiling was continued for $10 \mathrm{~min}$; the heating mantle was then removed, and stirring was continued for an additional $15 \mathrm{~min}$. After the solution cooled to room temperature it was stored at $4^{\circ} \mathrm{C}$.

\subsection{Preparation of biotin-MB-AuNP biolabel}

The biotin-MB-AuNP conjugate was synthesised in accordance with a previously published protocol [22]. Briefly; $250 \mu \mathrm{L}$ of the AuNPs (OD 2.3) in $0.1 \mathrm{mM}$ phosphate buffer at $\mathrm{pH} 7.4$ were mixed, in a $\mathrm{NaOH}$ treated glass vial, with adequate volume of the MB stock solution to obtain a final DNA-to-AuNPs ratio of 500:1. The solution was then left to react at room temperature, under gentle mixing overnight. The biotin-MB-AuNP mixture was finally subjected to an "aging process" consisting of a stepwise increase of the concentration of $\mathrm{NaCl}$ up to $0.3 \mathrm{M}$; this was followed by an overnight incubation at room temperature under gentle shaking. Finally, the biotin-MB-AuNP conjugates were washed twice by sequential centrifugation $\left(24,000 \mathrm{~g}, 20 \mathrm{~min}, 20^{\circ} \mathrm{C}\right)$, resuspension in $\mathrm{NaCl} 0.3 \mathrm{M}$ and $0.1 \mathrm{mM}$ phosphate buffer, $\mathrm{pH} 7.4$ and stored at $4^{\circ} \mathrm{C}$ until use.

\subsection{Fabrication of neutravidin electrode}

A glassy carbon electrode (GCE) was first polished with 0.3 and $0.05 \mu \mathrm{m}$ alumina and then sonicated by ultrasonic cleaning in ethanol and ultrapure water. The clean electrode was dried with high-purity nitrogen. A $5 \mu \mathrm{L}$ aliquot of $0.25 \mathrm{mg} / \mathrm{mL}$ neutravidin solution was drop-cast onto the clean glassy carbon electrode, and the electrode was kept dry at room temperature for 1h. The modified electrode was cross-linked with glutaraldehyde vapour ( $25 \%$ in water on a hot plate at $40{ }^{\circ} \mathrm{C}$ ) for 45 minutes and then the electrodes were washed with PBS buffer, dried

\subsection{Detection of miRNA-21}

Detection of the target miRNA was performed by incubating the neutravidin modified glassy carbon working electrodes in the solution containing miRNA-21 in the presence of the biotinMB-AuNP label. The solution was prepared by mixing a desired amount of target miRNA with 
an optimised concentration of biotin-MB-AuNP in a buffer solution (10 $\mathrm{mM}$ phosphate buffer $\mathrm{pH}$; PB containing $500 \mathrm{mM} \mathrm{NaCl}, \mathrm{pH}=7.5$ ) to a final volume of $25 \mu \mathrm{L}$. After incubation, the electrode was rinsed with $25 \mu \mathrm{L}$ of $10 \mathrm{mM}$ phosphate buffer solution ( $\mathrm{pH} 7.4$ ), and subsequently detected by SSWV.

\subsection{Silver enhancement}

After hybridisation, the modified electrode was incubated in the silver enhancer solution (equal volumes $(200 \mu \mathrm{L})$ of each solutions $\mathrm{A}$ and $\mathrm{B}$ from the silver enhancer kit were mixed together) to enhance the electrochemical signal intensity of gold nanoparticles by forming shells of silver around of them. After $10 \mathrm{~min}$, the solution was removed and sodium thiosulfate $(0.2$ M) was then dropped on the surface for 2 min to stop the silver reaction. Finally, the electrode was thoroughly washed with double-distilled water [23].

\section{Results and Discussion}

\subsection{Design of the voltammetric miRNA biosensor}

Scheme 1 illustrates the working principle of the voltammetric miRNA biosensor. The signal generation mechanism is based on a series of simultaneous events within a one-pot reaction by simple addition of miRNA-21/biotin-MB-AuNPs mixture onto the neutravidin modified sensor surface. In the presence of target miRNA-21, hybridisation takes place resulting in the opening of the biotin-MBs. This makes the biotin group that was previously "protected" by the steric hindrance of the stem-loop structure, accessible. The activated biotinMB-AuNP/miRNA complexes were then available for capture, via supramolecular interaction, onto a neutravidin modified glassy carbon electrode. The binding event resulted in an increase in the peak current at the working electrode/electrolyte interface. The analytical performance of the biosensor was improved by silver deposition onto the captured AuNPs. In the absence of target miRNA-21, the biotin-MB retains its stem-loop structure, thus sterically impeding the interaction between the biotin group and the neutravidin on the electrode surface.

This approach combines the advantages of a dual-functional biolabel and a simple neutravidin electrode for quantitative electrochemical detection of miRNA-21. The concept of using a neutravidin electrode for miRNA detection provides a more stable and robust sensor platform compared with conventional nucleic biosensors based on immobilised single-stranded DNA probes. Moreover, the use of the MB approach is important for detection of the short length miRNA, while the conventional sandwich hybridisation approach is difficult to apply for detection of miRNA, since it is limited by the length of the single-stranded DNA probe. 


\subsection{Characterisation of the biotin-MB-AuNP label}

203

204

205

206

207

208

209

210

211

212

213

To follow the steps of biofunctionalisation, dynamic light scattering measurements were used to determine the size of the synthesised citrate-capped AuNPs, biotin-MB-AuNP label and biotin-MB-AuNP/miRNA-21 complexes.The average hydrodynamic diameters obtained were $29 \pm 0.6,40.3 \pm 1.9$ and $55.3 \pm 2.4 \mathrm{~nm}$, respectively. An increase in hydrodynamic diameter was observed for the biotin-MB-AuNPs compared with the AuNPs; this indicates the successful immobilisation of the MB onto the AuNPs. A further increase in the hydrodynamic diameter was recorded after formation of the biotin-MB-AuNP/miRNA-21 complex, indicating the opening of the MB stem-loop structure.

Zeta potential measurements were performed to study the surface charge densities of the AuNPs, biotin-MB-AuNP label and biotin-MB-AuNP/miRNA-21 complex. Following functionalisation of the AuNPs with MBs, an increase in the zeta potential was recorded from $-27.3 \pm 2.5 \mathrm{mV}$ (AuNPs) to $-35.3 \pm 1.4 \mathrm{mV}$ (MB-AuNPs). After hybridisation with target miRNA-21, the zeta potential of the biotin-MB-AuNPs/miRNA-21 complex further increased to $-42.3 \pm 2.6 \mathrm{mV}$. These increases in zeta potential values are related to the high negative charges of the MBs and miRNAs.

\subsection{Optimisations of the voltammetric miRNA biosensor}

In order to improve the performance of the biosensor, optimisation of: (i) the pretreatment time for dissolution of AuNPs into Au ions; (ii) the applied potential during the pretreatment; (iii) the concentration of biotin-MB-AuNPs label; and (iv) the amount of immobilised neutravidin was performed.

SSWV was employed to study the influence of time and potential during the pretreatment on the oxidation peak current of miRNA-21. It is known that an increase in the pretreatment time improves the sensitivity of determination [24]. Hence, the effect of variation of the time was studied over a period of (10,30,60,120, 180, and $240 \mathrm{~s})$, employing a frequency of $20 \mathrm{~Hz}$ and a potential of $1 \mathrm{~V}$. Figure $1 \mathrm{~A}$ shows that the signal response increased with increasing time. Therefore, 180 min was chosen as the preferred time, since it proved a good compromise between the assay time and the signal response.

Keeping the frequency as $20 \mathrm{~Hz}$ and the time of pretreatment as $180 \mathrm{~s}$, the optimum potential was determined by scanning a potential window of 0.5 to $1.25 \mathrm{~V}$. As can be seen from Figure 1B, the peak current for detection of $0.5 \times 10^{-9} \mathrm{M}$ miRNA-21 reached its maximum at a potential of $1.25 \mathrm{~V}$, but the transition from 1 to $1.25 \mathrm{~V}$ did not significantly influence the sensor 
response. Thus, the potential during the pre-treatment was fixed at $1 \mathrm{~V}$ for further studies, because a higher potential may damage the electrode surface. The final conditions for the SSWV therefore were $\mathrm{E}=1 \mathrm{~V}, \mathrm{t}=180 \mathrm{~s}$ and $\mathrm{f}=20 \mathrm{~Hz}$.

The effect of the amount of biotin-MB-AuNP label on the biosensor performance was investigated by detecting $0.5 \times 10^{-9} \mathrm{M}$ of the target miRNA-21 using different concentration ( 1.56 to $3.68 \mathrm{mg} / \mathrm{mL}$ ) of biotin-MB-AuNPs. The peak current increased with increasing amount of biotin-MB-AuNPs from 1.56 to $3.12 \mathrm{mg} / \mathrm{mL}$. When the concentration of the biotin-MBAuNPs further increased from 3.12 to $3.68 \mathrm{mg} / \mathrm{mL}$, the peak current decreased slightly, probably due to the use of higher concentrations of the biotin-MB-AuNPs, which seems to have a saturation effect on the signal response [22, 25]. Subsequently, $3.12 \mathrm{mg} / \mathrm{mL}$ of the biotin-MBAuNP was used as the optimised concentration. (Figure 1C).

To define the nature of the capturing layer, optimisation of the amount of neutravidin on the transducer surface was performed by preparing electrodes with various neutravidin concentrations $(0.15,0.25,0.5$ and $1 \mathrm{mg} / \mathrm{mL})$. The responses of the sensors to $0.5 \times 10^{-9} \mathrm{M}$ of target miRNA-21 are presented in Figure 1D. A closer analysis showed that the normalised signal for the detection of $0.5 \times 10^{-9} \mathrm{M}$ miRNA-21increased with decreasing amount of the neutravidin on the electrode surface from 1.0 to $0.25 \mathrm{mg} / \mathrm{mL}$, while a further a further decrease in neutravidin concentration to $0.15 \mathrm{mg} / \mathrm{mL}$ delivered insignificant improvement to the signal response. Therefore, $0.25 \mathrm{mg} / \mathrm{mL}$ of neutravidin was used for preparation of the biosensors.

\subsection{Sensitivity and selectivity of the miRNA biosensor}

The analytical performance of the electrochemical biosensor was investigated under the optimised experimental conditions. After performing the affinity assay step, the electrode was immersed into a measuring cell containing $0.1 \mathrm{M}$ sulfuric acid. The electrochemical oxidation of AuNPs to $\mathrm{Au}^{3+}$ ions was performed at $+1 \mathrm{~V} v s \mathrm{Ag} / \mathrm{AgCl}$ for $180 \mathrm{~s}$ in a unstirred solution. Immediately after the electrochemical oxidation step, striping square wave voltammetry (SSWV) was performed. During this step the potential was scanned from +1.0 to $0 \mathrm{~V}$ (step potential $=10 \mathrm{mV}$, frequency $=20 \mathrm{~Hz}$ ), resulting in an analytical signal due to the reduction of $\mathrm{Au}$ ions. The modified GCE displayed a well-defined response at $+0.84 \mathrm{~V} v s \mathrm{Ag} / \mathrm{AgCl}$ to $\mathrm{Au}^{3+}$. The detection peak corresponded to the reduction of the $\mathrm{Au}^{3+}$ to $\mathrm{Au}^{0}$ on the surface of GCE.

The SSWV peak currents rose significantly with increasing concentrations of target miRNA-21 (insert Figure 2). A good linear relationship between the peak currents and the miRNA-2 1 concentration was achieved in range of $0.5 \times 10^{-12}$ to $1.0 \times 10^{-9} \mathrm{M}$ with a coefficient of determination $\left(\mathrm{R}^{2}\right)$ of 0.985 and the limit of detection (LOD) was calculated to be $0.1 \times 10^{-12}$ 


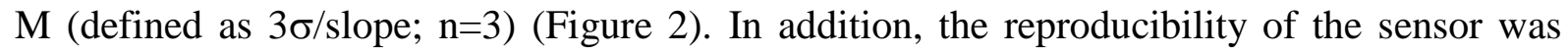
further investigated by measuring the target miRNA-21 at $50 \times 10^{-12}$ and $0.5 \times 10^{-9} \mathrm{M}$ with five replicates. The coefficients of variation for both measured concentrations were $4.2 \%$ and $5.5 \%$, respectively (Figure S1). Thus, the biosensor displayed high sensitivity and acceptable reproducibility, and could be applied to quantification of miRNA-21 at low concentrations.

The selectivity of the biosensor was evaluated by analysing closely related miRNAs (miRNA-221 and miRNA-205), which are members of the same miRNA family and have both been associated with cancers: miRNA-205 in breast [26], prostate [27], lung [28] and bladder [29] cancers; and miRNA-221 in bladder [30] and as trocytic tumours [31]. Figure 3 shows that a clear response, was observed in the presence of target miRNA-21 $\left(0.5 \times 10^{-12} \mathrm{M}\right)$ while no significant signal was observed for either miRNA-205 (with $41 \%$ similarity) or formiRNA-221 (with $18 \%$ similarity), even at significantly higher concentration $\left(1 \times 10^{-9} \mathrm{M}\right)$. Interestingly, we did not observe a significant influence of miRNA-21, when this was mixed in large excess (1000 fold) with the other non-targeted miRNAs. It should be noted that miRNAs are specific biomarkers present in blood stream and do not offer the possibility for single-base mismatch detection associated with genetic analysis of nucleic acids. Moreover, the non-target miRNA205 chosen is one of the miRNAs with the highest similarity to miRNA-21.

\subsection{Analytical performance of the silver enhanced miRNA biosensor}

Enhancement by precipitation of silver onto AuNP labels have been reported to achieve amplified signals and lower detection limits [32,33]. Silver enhancement technology, using voltammetry techniques, has been previously reported [23]. After the affinity capture of the miRNA-21/biotin-MB-AuNP complex onto the electrode surface, the electrode was incubated in a silver enhancement solution composed of silver ions. The AuNPs act as a catalyst and reduce silver ions into metallic silver in the presence of a reducing agent. The reduced metallic silver deposits on the AuNP surfaces, thus forming an enlarged metallic silver cluster around the AuNPs. Subsequently, silver deposition time followed by voltammetric detection were optimised in order to maximise the sensitivity and to shorten the assay time. Figure 4 shows the effect of the silver deposition time on the SSWV signal response for $0.5 \times 10^{-9} \mathrm{M}$ of miRNA-21. The peak current signal increased against the silver deposition time of 2 to $12 \mathrm{~min}$. Ten minutes was chosen as the preferred time since it proved a good compromise between the silver deposition time and the signal response.

The analytical performance of the silver enhanced miRNA biosensors was investigated by varying the target miRNA-21 concentration. After the silver enhancement step, the electrode 
was immersed in a measuring cell containing $10 \mathrm{mM}$ nitric acid. Nitric acid is required for the efficient oxidative dissolution of metallic silver. The electrochemical oxidation of metallic silver to $\mathrm{Ag}^{1+}$ ions was performed at $+1 \mathrm{~V} v s . \mathrm{Ag} / \mathrm{AgCl}$ for $180 \mathrm{~s}$ in the non-stirred solution. Immediately after the electrochemical oxidation step, striping square wave voltammetry (SSWV) was performed. During this step the potential was scanned from 0 to $0.8 \mathrm{~V}$ (step potential $=10 \mathrm{mV}$, frequency $=20 \mathrm{~Hz}$ ), resulting in an analytical signal due to the oxidation of $\mathrm{Ag}^{1+}$. The modified GCE displayed a well-defined response at $+0.27 \mathrm{~V} v$ s. $\mathrm{Ag} / \mathrm{AgCl}$ to $\mathrm{Ag}^{1+}$. The detection peak corresponded to the oxidation of the $\mathrm{Ag}^{1+}$ to $\mathrm{Ag}^{2+}$ on the surface of GCE.

Experiments showed that the signal obtained from the oxidation peak current of silver could detect miRNA-21 over an extremely wide dynamic range from $10 \times 10^{-15}$ to $1.0 \times 10^{-9} \mathrm{M}$ covering 5 orders of magnitude of miRNA concentration (Figure 5), where the lowest detectable miRNA-21 concentration was significantly improved by 50 times compared to measurements without silver enhancement. The regression equation of the miRNA biosensor between miRNA concentration of $1.0 \times 10^{-10}$ to $5.0 \times 10^{-9} \mathrm{M}$ was $Y=1.62 X+3.47$ (where $\mathrm{X}$ is the logarithm of target miRNA-21 concentration (M); and $\mathrm{Y}$ is the SSWV peak current $(\mu \mathrm{A})$ ) with a coefficient of determination $\left(\mathrm{R}^{2}\right)$ of 0.995 , and the limit of detection (LOD) was calculated to be $4.0 \times 10^{-}$ ${ }^{15} \mathrm{M}$ (defined as $3 \sigma /$ slope; $\mathrm{n}=3$ ).

A selectivity study of the biosensor was performed after silver enhancement by analysing closely related miRNAs (miRNA-221 and miRNA-205). Figure 6 shows that a clear response, was observed in the presence of target miRNA-21 $\left(10 \times 10^{-15} \mathrm{M}\right)$ while no significant signal was observed for either miRNA-205 or formiRNA-221, even at significantly higher concentration $\left(1 \times 10^{-9} \mathrm{M}\right)$. Interestingly, we did not observe a significant influence of miRNA21, when this was mixed in large excess with the other non-target miRNAs. This result confirms that the addition of silver enhancement did not significantly influence the selectivity of the miRNA-21 biosensors.

\subsection{Detection of miRNA-21 in spiked serum sample}

Detection of miRNA-21 in spiked human serum samples were investigated using the standard addition method. Serum samples with miRNA-21 concentrations of $50 \times 10^{-15}, 500 \times$ $10^{-15}, 1.0 \times 10^{-12}$ and $100 \times 10^{-12} \mathrm{M}$ were prepared and measured. Serum sample with a background level of miRNA-21 equal to $50 \times 10^{-15} \mathrm{M}$ was incubated in the biosensor and the signal fitted to the calibration curve in Figure 5 to calculate an approximate concentration value. After this, solutions containing approximately twice and three times the calculated preliminary concentration were prepared by spiking the sample with adequate volumes of a miRNA-21 
stock solution. The responses obtained for the sample and for the spiked solutions were then plotted and miRNA-21 concentration in sample was calculated by extrapolating the linear curve, obtained by plotting the responses vs the nominal concentration of added stock miRNA21. The measured and the actual concentration of miRNA-21 in the spiked serum samples are compared in Table 1 and the correlation plot (measured concentration using the standard addition method $v s$ the actual concentration) is shown in Figure 7. As it can be seen from Table 1 and Figure 7, there was a good match between the measured experimental values and the nominal concentration of the miRNA-21 in the serum samples (slope of $0.93 ; \mathrm{R}^{2}=0.997$ ). Thus, the developed biosensor allowed accurate detection of miRNA-21 not only in buffer solutions, but also in spiked serum samples.

\section{Conclusion}

A simple and highly sensitive neutravidin-based biosensor combined with a dual-functional gold nanoparticle (AuNP) biolabel have been developed for microRNA-21 detection. The dual functional probe was comprised of AuNPs coupled with a biotinylated molecular beacon (biotin-MB) for both biorecognition and signal generation. Although the concept of the MBAuNP label system has been demonstrated with great success for solid-phase affinity bioassays for rapid and simple visual detection of various analytes, it only provided semi-quantitative results and suffered from relatively low sensitivity. We report for the first time the combination of such a dual-functional biolabel for quantitative electrochemical detection of miRNA. In the presence of target miRNA-21, hybridisation takes place resulting in the "activation" of the biotin-MB; this event makes the biotin group, which was previously "protected" by the steric hindrance of the MB stem-loop structure, accessible. Simultaneously, the activated biotin-MBAuNPs/miRNA complexes become available for capture, via supramolecular interaction onto a neutravidin-modified electrode, for electrochemical transduction within a single step. The captured AuNPs were detected by stripping square wave voltammetry. The performance of the miRNA biosensor was further improved by silver enhancement delivering a detection limit of $4.0 \times 10^{-15} \mathrm{M}$ miRNA-21, and an extremely wide analytic dynamic range from $10 \times 10^{-15}$ to $1.0 \times 10^{-9} \mathrm{M}$ (5 orders of magnitude). The biosensor offers a combination of simple operation, high sensitivity and wide analytical range for the detection of miRNA and could provide a powerful and convenient tool for biomedical research and applications in cancer diagnostics.

\section{Acknowledgement}


370 This work was partially funded by the "SMARTCANCERSENS" project from the European

371 Communities Seventh Framework Program under the Grant Agreement PIRSES-GA-2012372318053. 


\section{References:}

[1] R.C. Lee, R.L. Feinbaum, V. Ambros, The C. elegans heterochronic gene lin-4 encodes small RNAs with antisense complementarity to lin-14, Cell. 75 (1993) 843-854.

[2] S. Griffiths-Jones, The microRNA Registry, Nucleic Acids Res. 32 (2004) D109-111.

[3] J.S. Mattick, I.V. Makunin, Non-coding RNA, Hum. Mol. Genet. 15 (2006) R17-R29.

[4] E. Wienholds, W.P. Kloosterman, E. Miska, E. Alvarez-Saavedra, E. Berezikov, E. de Bruijn, H.R. Horvitz, S. Kauppinen, R.H.A. Plasterk, MicroRNA expression in zebrafish embryonic development, Science. 309 (2005) 310-311.

[5] C.M. Croce, Causes and consequences of microRNA dysregulation in cancer, Nat. Rev. Genet. 10 (2009) 704-714.

[6] L.A. Torre, F. Bray, R.L. Siegel, J. Ferlay, J. Lortet-Tieulent, A. Jemal, Global cancer statistics, 2012, CA. Cancer J. Clin. 65 (2015) 87-108.

[7] M.C.S. Wong, W.B. Goggins, H.H.X. Wang, F.D.H. Fung, C. Leung, S.Y.S. Wong, C.F. Ng, J.J.Y. Sung, Global Incidence and Mortality for Prostate Cancer: Analysis of Temporal Patterns and Trends in 36 Countries, Eur. Urol. (2016).

[8] M.M. Center, A. Jemal, J. Lortet-Tieulent, E. Ward, J. Ferlay, O. Brawley, F. Bray, International variation in prostate cancer incidence and mortality rates, Eur. Urol. 61 (2012) 1079-1092.

[9] E.A. Hunt, A.M. Goulding, S.K. Deo, Direct detection and quantification of microRNAs, Anal. Biochem. 387 (2009) 1-12.

[10] M. de Planell-Saguer, M.C. Rodicio, Analytical aspects of microRNA in diagnostics: A review, Anal. Chim. Acta. 699 (2011) 134-152.

[11] E. Paleček, M. Bartošík, Electrochemistry of Nucleic Acids, Chem. Rev. 112 (2012) 3427-3481.

[12] A. Gao, N. Lu, P. Dai, T. Li, Y. Wang, Label-free and ultrasensitive detection of microrna biomarkers in lung cancer cells based on silicon nanowire FET biosensors, in: 2013 Transducers Eurosensors XXVII 17th Int. Conf. Solid-State Sens. Actuators Microsyst. TRANSDUCERS EUROSENSORS XXVII, 2013: pp. 2439-2442.

[13] Y. Peng, G. Yi, Z. Gao, A highly sensitive microRNA biosensor based on ruthenium oxide nanoparticle-initiated polymerization of aniline, Chem. Commun. 46 (2010) 9131-9133.

[14] H. Yin, Y. Zhou, H. Zhang, X. Meng, S. Ai, Electrochemical determination of microRNA-21 based on graphene, LNA integrated molecular beacon, AuNPs and biotin multifunctional bio bar codes and enzymatic assay system, Biosens. Bioelectron. 33 (2012) 247-253.

[15] F. Bettazzi, E. Hamid-Asl, C.L. Esposito, C. Quintavalle, N. Formisano, S. Laschi, S. Catuogno, M. Iaboni, G. Marrazza, M. Mascini, L. Cerchia, V.D. Franciscis, G. Condorelli, I. Palchetti, Electrochemical detection of miRNA-222 by use of a magnetic bead-based bioassay, Anal. Bioanal. Chem. 405 (2012) 1025-1034.

[16] Y. Peng, Z. Gao, Amplified detection of microRNA based on ruthenium oxide nanoparticle-initiated deposition of an insulating film, Anal. Chem. 83 (2011) 820-827.

[17] T. Kilic, S.N. Topkaya, D. Ozkan Ariksoysal, M. Ozsoz, P. Ballar, Y. Erac, O. Gozen, Electrochemical based detection of microRNA, mir21 in breast cancer cells, Biosens. Bioelectron. 38 (2012) 195-201.

[18] H. Dong, S. Jin, H. Ju, K. Hao, L.-P. Xu, H. Lu, X. Zhang, Trace and Label-Free MicroRNA Detection Using Oligonucleotide Encapsulated Silver Nanoclusters as Probes, Anal. Chem. 84 (2012) 8670-8674.

[19] M. Bartosik, R. Hrstka, E. Palecek, B. Vojtesek, Magnetic bead-based hybridization assay for electrochemical detection of microRNA, Anal. Chim. Acta. 813 (2014) 3540. 
[20] Y. Zhou, M. Wang, Z. Xu, C. Ni, H. Yin, S. Ai, Investigation of the effect of phytohormone on the expression of microRNA-159a in Arabidopsis thaliana seedlings based on mimic enzyme catalysis systematic electrochemical biosensor, Biosens. Bioelectron. 54 (2014) 244-250.

[21] L. Liu, N. Xia, H. Liu, X. Kang, X. Liu, C. Xue, X. He, Highly sensitive and label-free electrochemical detection of microRNAs based on triple signal amplification of multifunctional gold nanoparticles, enzymes and redox-cycling reaction, Biosens. Bioelectron. 53 (2014) 399-405.

[22] K. Kor, A.P.F. Turner, K. Zarei, M. Atabati, V. Beni, W.C. Mak, Structurally responsive oligonucleotide-based single-probe lateral-flow test for detection of miRNA-21 mimics, Anal. Bioanal. Chem. 408 (2016) 1475-1485.

[23] H. Cai, Y. Wang, P. He, Y. Fang, Electrochemical detection of DNA hybridization based on silver-enhanced gold nanoparticle label, Anal. Chim. Acta. 469 (2002) 165172.

[24] B.J. Sanghavi, A.K. Srivastava, Simultaneous voltammetric determination of acetaminophen and tramadol using Dowex50wx2 and gold nanoparticles modified glassy carbon paste electrode, Anal. Chim. Acta. 706 (2011) 246-254.

[25] Y. Peng, J. Jiang, R. Yu, A sensitive electrochemical biosensor for microRNA detection based on streptavidin-gold nanoparticles and enzymatic amplification, Anal. Methods. 6 (2014) 2889-2893.

[26] S.B. Greene, J.I. Herschkowitz, J.M. Rosen, The ups and downs of miR-205: identifying the roles of miR-205 in mammary gland development and breast cancer, RNA Biol. 7 (2010) 300-304.

[27] S. Majid, A.A. Dar, S. Saini, S. Yamamura, H. Hirata, Y. Tanaka, G. Deng, R. Dahiya, MicroRNA-205-directed transcriptional activation of tumor suppressor genes in prostate cancer, Cancer. 116 (2010) 5637-5649.

[28] A. Markou, E.G. Tsaroucha, L. Kaklamanis, M. Fotinou, V. Georgoulias, E.S. Lianidou, Prognostic value of mature microRNA-21 and microRNA-205 overexpression in nonsmall cell lung cancer by quantitative real-time RT-PCR, Clin. Chem. 54 (2008) 16961704.

[29] E.D. Wiklund, J.B. Bramsen, T. Hulf, L. Dyrskjøt, R. Ramanathan, T.B. Hansen, S.B. Villadsen, S. Gao, M.S. Ostenfeld, M. Borre, M.E. Peter, T.F. Ørntoft, J. Kjems, S.J. Clark, Coordinated epigenetic repression of the miR-200 family and miR-205 in invasive bladder cancer, Int. J. Cancer J. Int. Cancer. 128 (2011) 1327-1334.

[30] Q. Lu, C. Lu, G.-P. Zhou, W. Zhang, H. Xiao, X.-R. Wang, MicroRNA-221 silencing predisposed human bladder cancer cells to undergo apoptosis induced by TRAIL, Urol. Oncol. 28 (2010) 635-641.

[31] A. Conti, M. Aguennouz, D. La Torre, C. Tomasello, S. Cardali, F.F. Angileri, F. Maio, A. Cama, A. Germanò, G. Vita, F. Tomasello, miR-21 and 221 upregulation and miR$181 \mathrm{~b}$ downregulation in human grade II-IV astrocytic tumors, J. Neurooncol. 93 (2009) 325-332.

[32] J. Wang, D. Xu, A.-N. Kawde, R. Polsky, Metal Nanoparticle-Based Electrochemical Stripping Potentiometric Detection of DNA Hybridization, Anal. Chem. 73 (2001) 5576-5581.

[33] T.M.-H. Lee, H. Cai, I.-M. Hsing, Effects of gold nanoparticle and electrode surface properties on electrocatalytic silver deposition for electrochemical DNA hybridization detection, The Analyst. 130 (2005) 364-369. 
Table 1. Actual and measured concentration of target miRNA-21 in spiked serum samples.

475

476

477

478

479

480

481

482

483

484

485

486

487

488

489

490

491

492

493

494

495

496

497

498

499

Scheme 1. A) Illustration of the design of the miRNA biosensor and B) the working principle of the neutravidin-based biosensor combined with a dual-functional gold nanoparticle (AuNP) probe for miRNA detection.

Figure 1. Optimisation of the miRNA biosensor: (A) the pretreatment time for stripping voltammetry $(\mathrm{E}=1 \mathrm{~V}$ and $\mathrm{f}=20 \mathrm{~Hz})$; $(\mathrm{B})$ the pretreatment potential $(\mathrm{t}=180 \mathrm{~s}$ and $\mathrm{f}=20 \mathrm{~Hz})$; (C) the amount of the biotin-MB-AuNPs biolabel; and (D) the amount of immobilised neutravidin onto GCE $(E=1 \mathrm{~V}, \mathrm{t}=180 \mathrm{~s}$ and $\mathrm{f}=20 \mathrm{~Hz})$.

Figure 2. Calibration curve shows the SSWV reduction peak current as a function of miRNA21 concentration $(n=3)$; (Insert) signal response curves of the biosensor obtained with various miRNA-21 concentrations $(\mathrm{E}=1 \mathrm{~V}, \mathrm{t}=180 \mathrm{~s}, \mathrm{f}=20 \mathrm{~Hz}$ and $0.1 \mathrm{M}$ sulphuric acid).

Figure 3. Selectivity studies of the biosensor for detection of target miRNA-21; non-specific miRNA-221 and miRNA-205; and miRNA-21 mixed with miRNA-221 and miRNA-205.

Figure 4. The blank and SSWV response of $1 \times 10^{-9} \mathrm{M}$ miRNA-21 in $10 \mathrm{mM}$ nitric acid with different silver deposition times.

Figure 5. Calibration curve shows the SSWV oxidation peak current after silver enhancement as a function of miRNA-21 concentration ( $n=3$ ); (Insert) signal response curves of the biosensor obtained with various miRNA-21 concentrations concentrations $(E=1 \mathrm{~V}, \mathrm{t}=180 \mathrm{~s}, \mathrm{f}=20 \mathrm{~Hz}$ and $10 \mathrm{mM}$ nitric acid). 
500 Figure 6. Selectivity studies of the biosensor after silver enhancement for detection of target 501 miRNA-21; non-specific miRNA-221 and miRNA-205; and miRNA-21 mixed with miRNA$502 \quad 221$ and miRNA-205.

503

504 Figure 7. A correlation plot between the measured and the actual concentration of miRNA-21 in spiked serum samples $(\mathrm{E}=1 \mathrm{~V}, \mathrm{t}=180 \mathrm{~s}, \mathrm{f}=20 \mathrm{~Hz}$ and $10 \mathrm{mM}$ nitric acid $)$. 
507

508 Table 1

\begin{tabular}{cc}
\hline Actual concentration & Measured concentration $(\mathbf{n}=3)$ \\
\hline $50 \times 10^{-15} \mathrm{M}$ & $(56 \pm 6.35) \times 10^{-15} \mathrm{M}$ \\
$500 \times 10^{-15} \mathrm{M}$ & $(577 \pm 34.2) \times 10^{-15} \mathrm{M}$ \\
$1.0 \times 10^{-12} \mathrm{M}$ & $(1.4 \pm 0.37) \times 10^{-12} \mathrm{M}$ \\
$100 \times 10^{-12} \mathrm{M}$ & $(108.5 \pm 5.7) \times 10^{-12} \mathrm{M}$ \\
\hline
\end{tabular}

509

510 


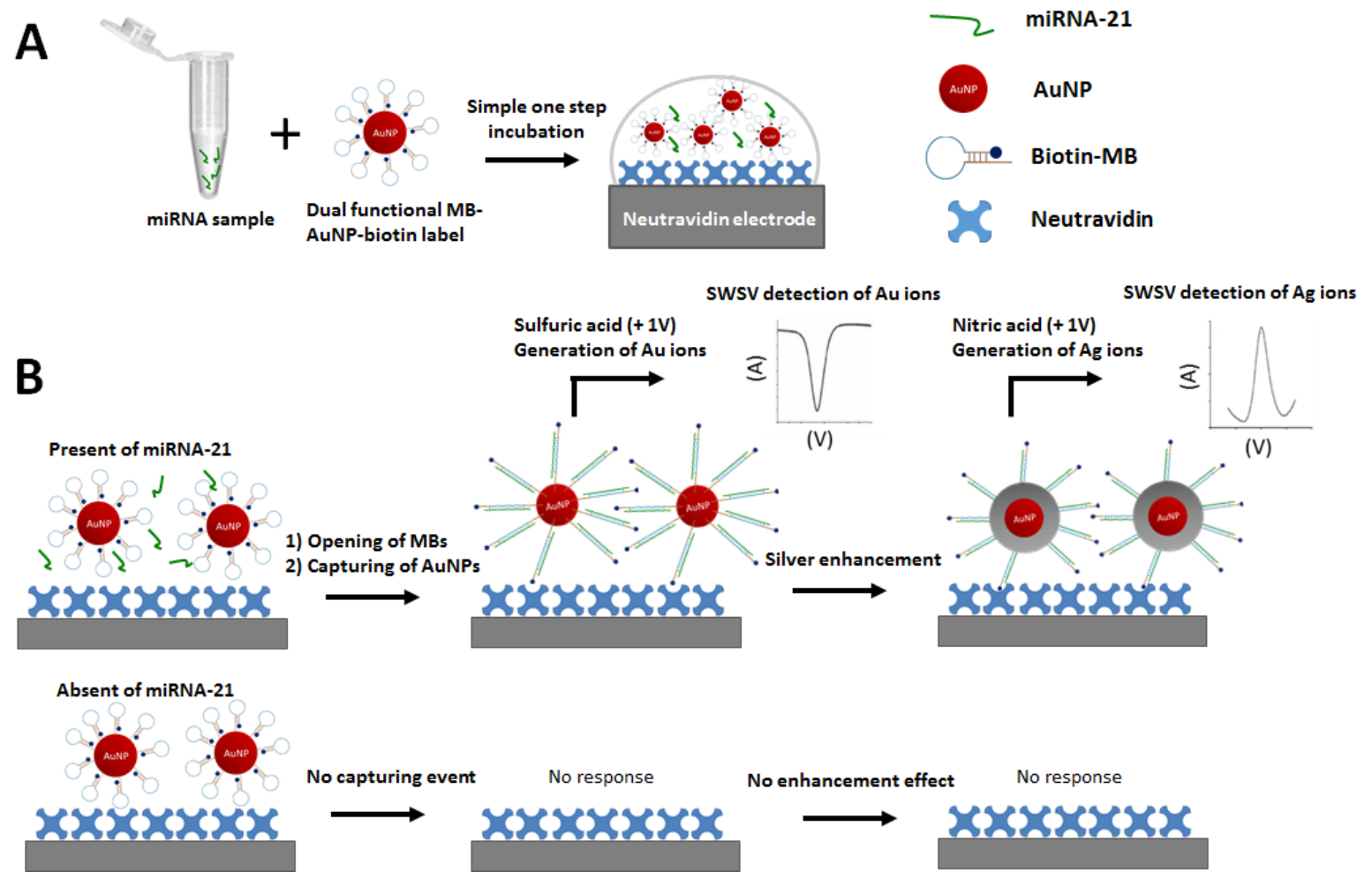

513

Scheme 1

515

516 

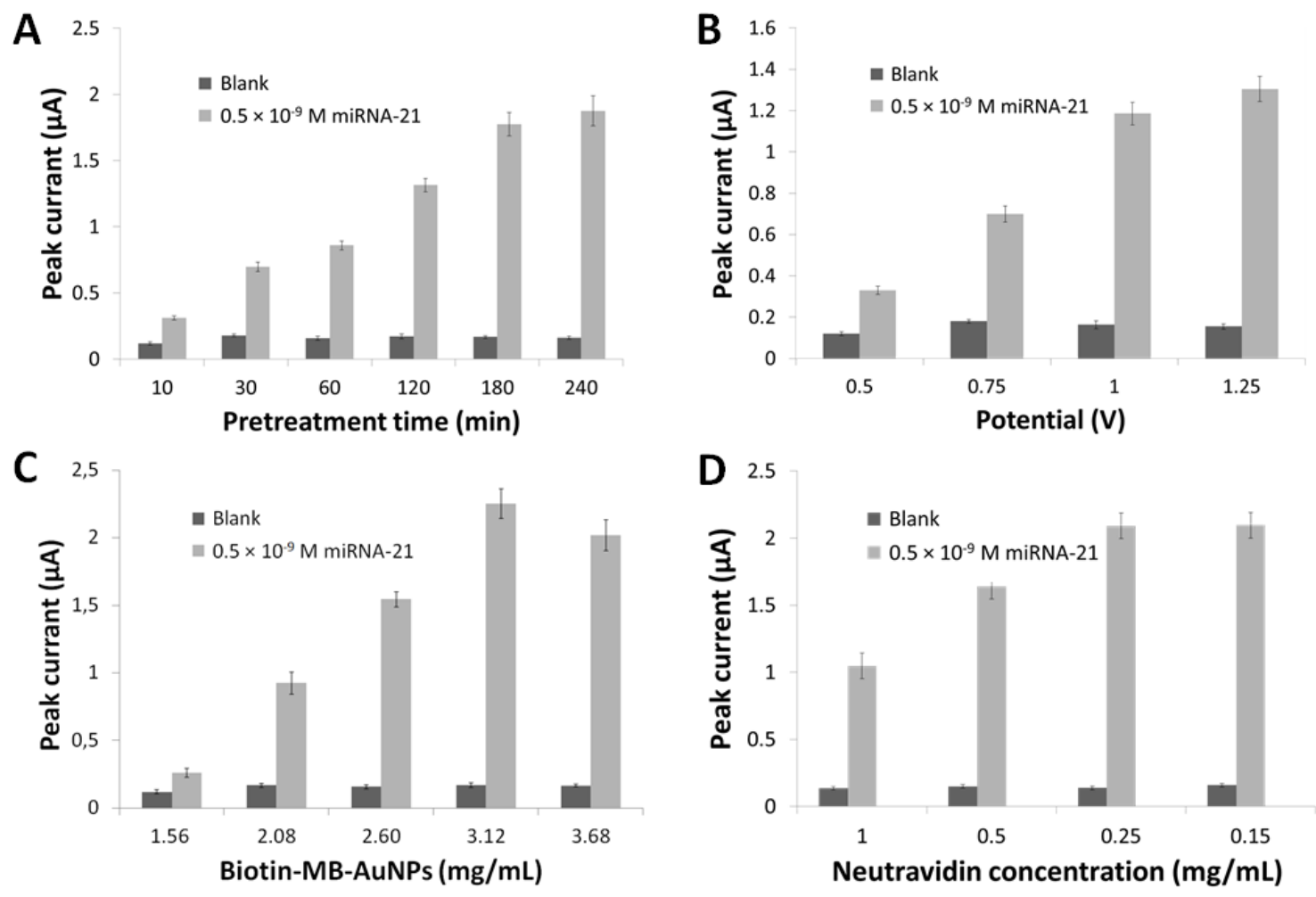

\section{Figure 1}




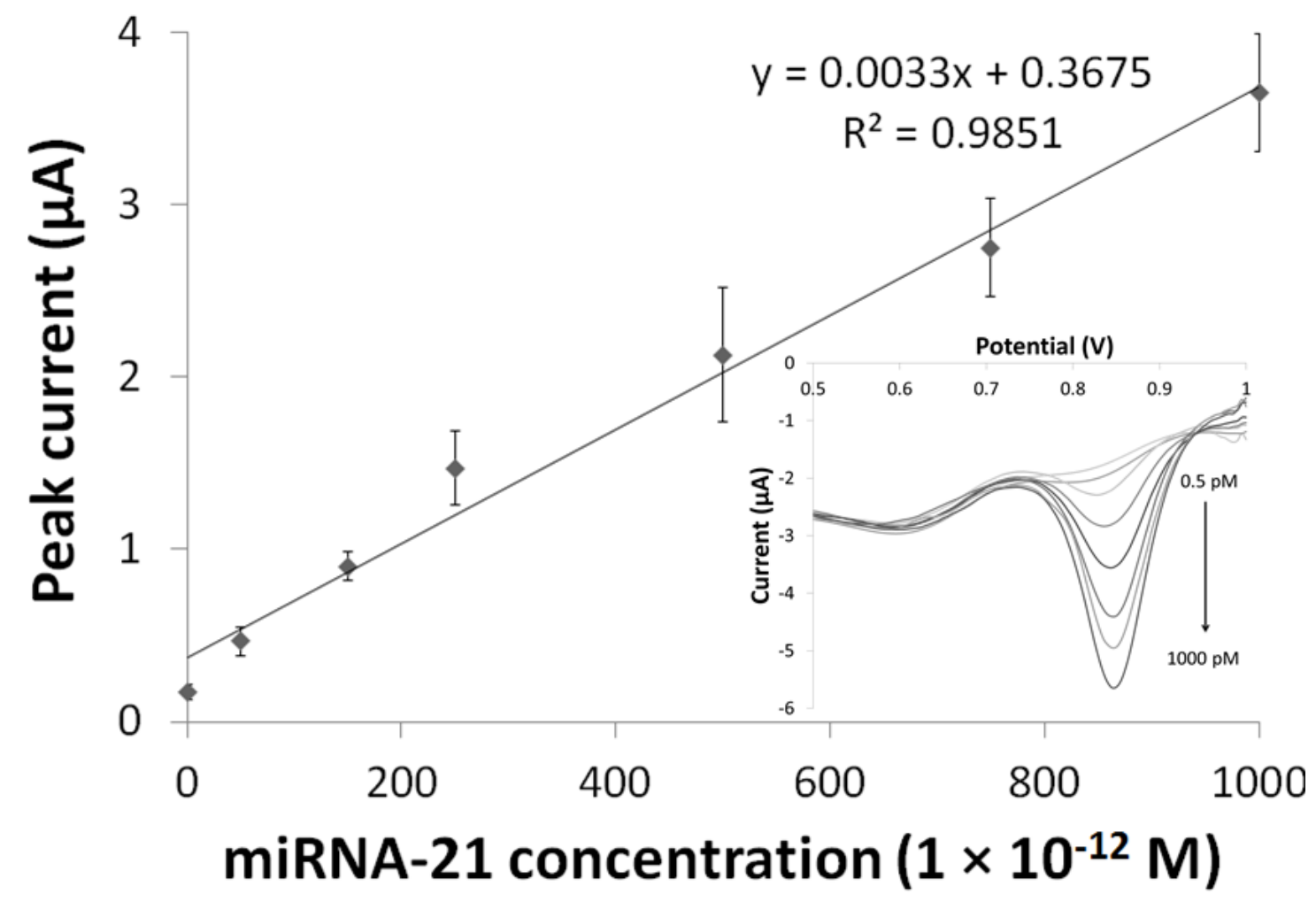

525

Figure 2

527

528

529 


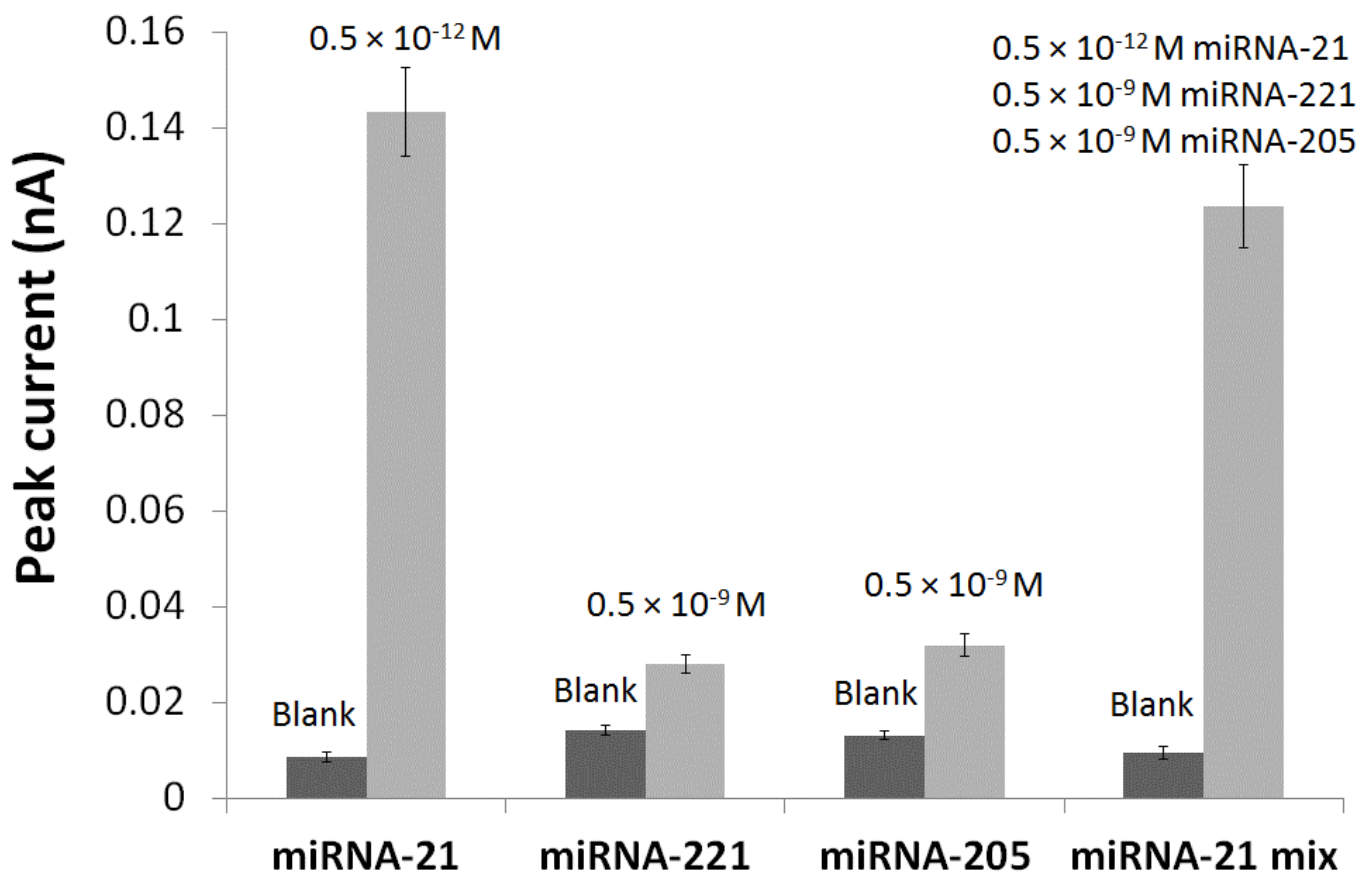

531

532

Figure 3

533

534 


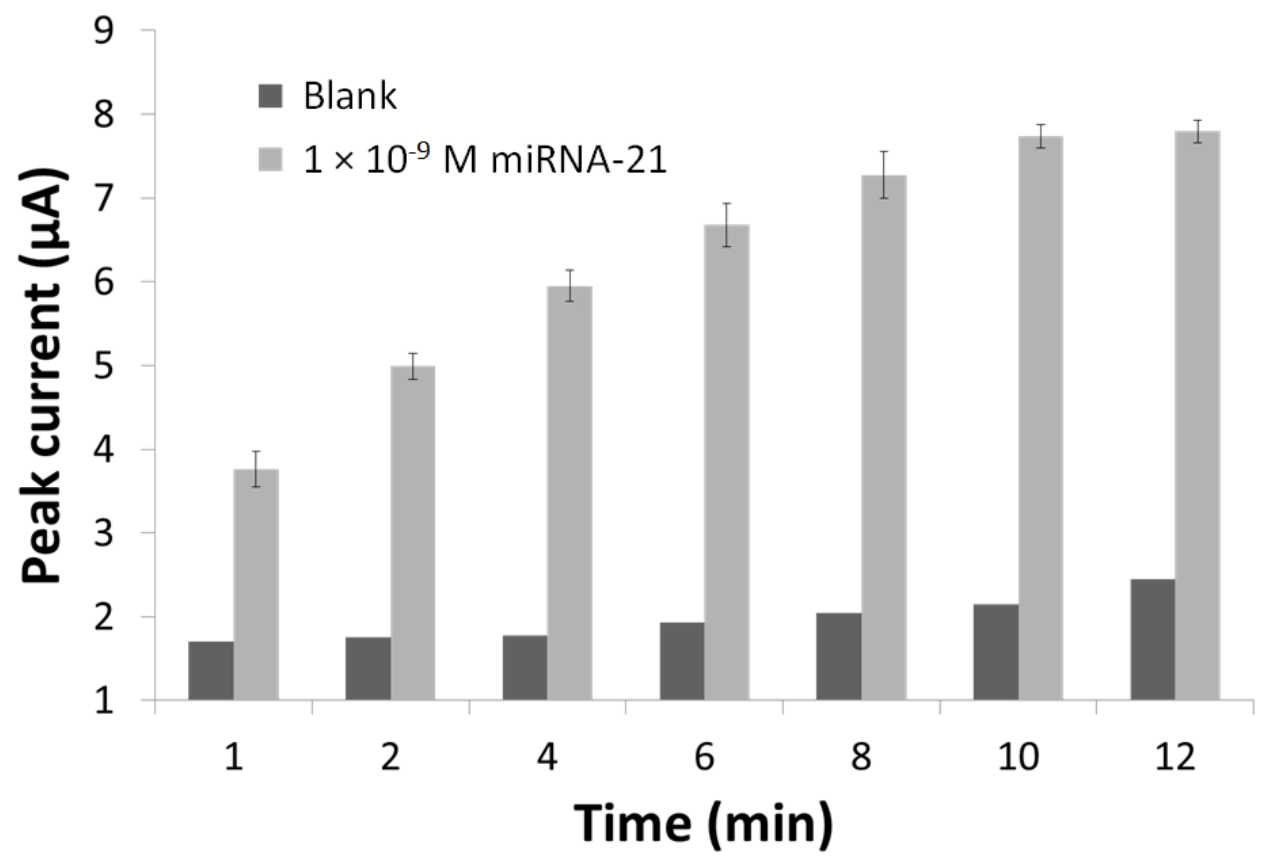

536

Figure 4

537

538

539

540

541 


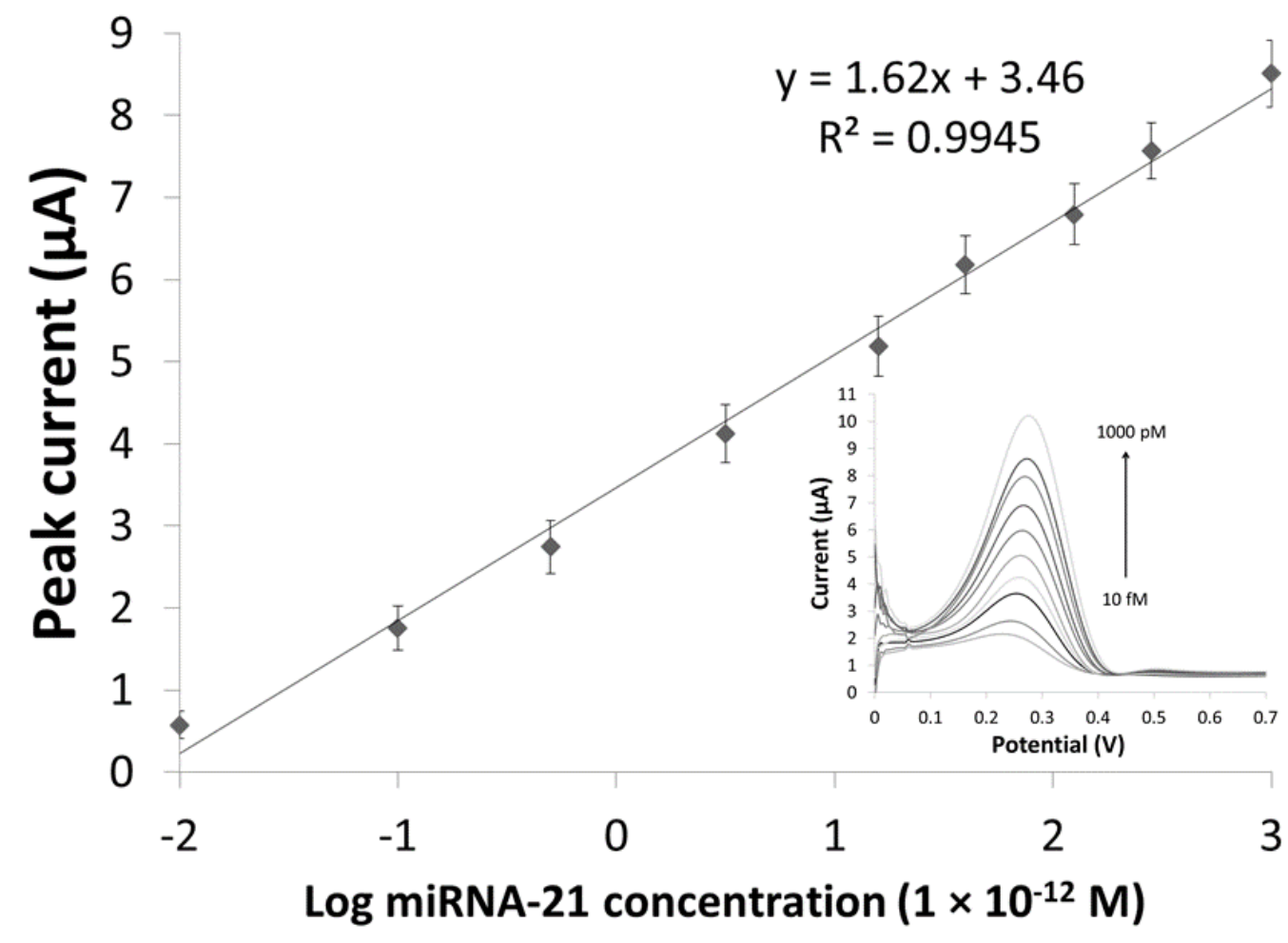

542

Figure 5

544

545

546

547

548 


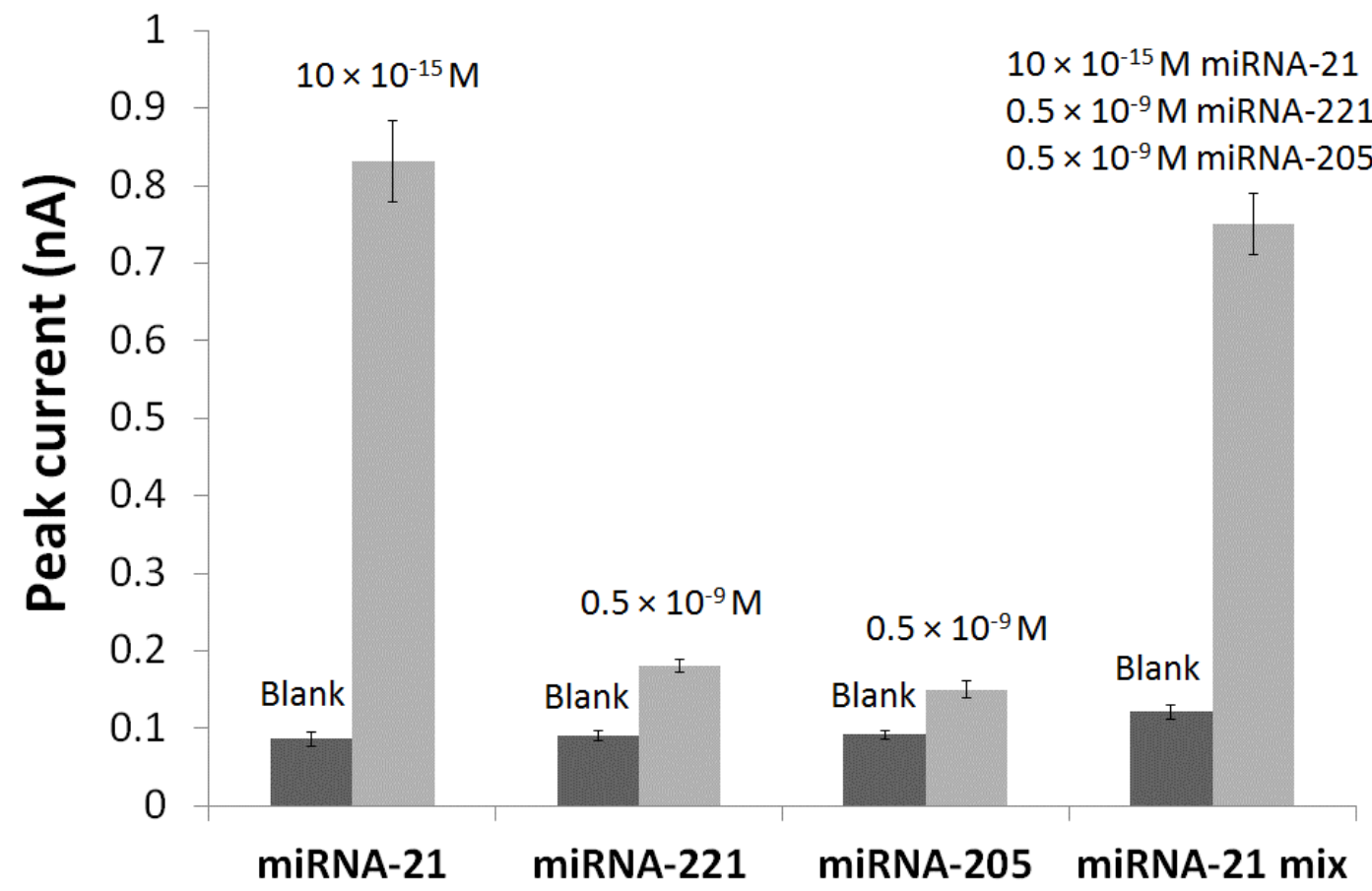

550

Figure 6

552

553 


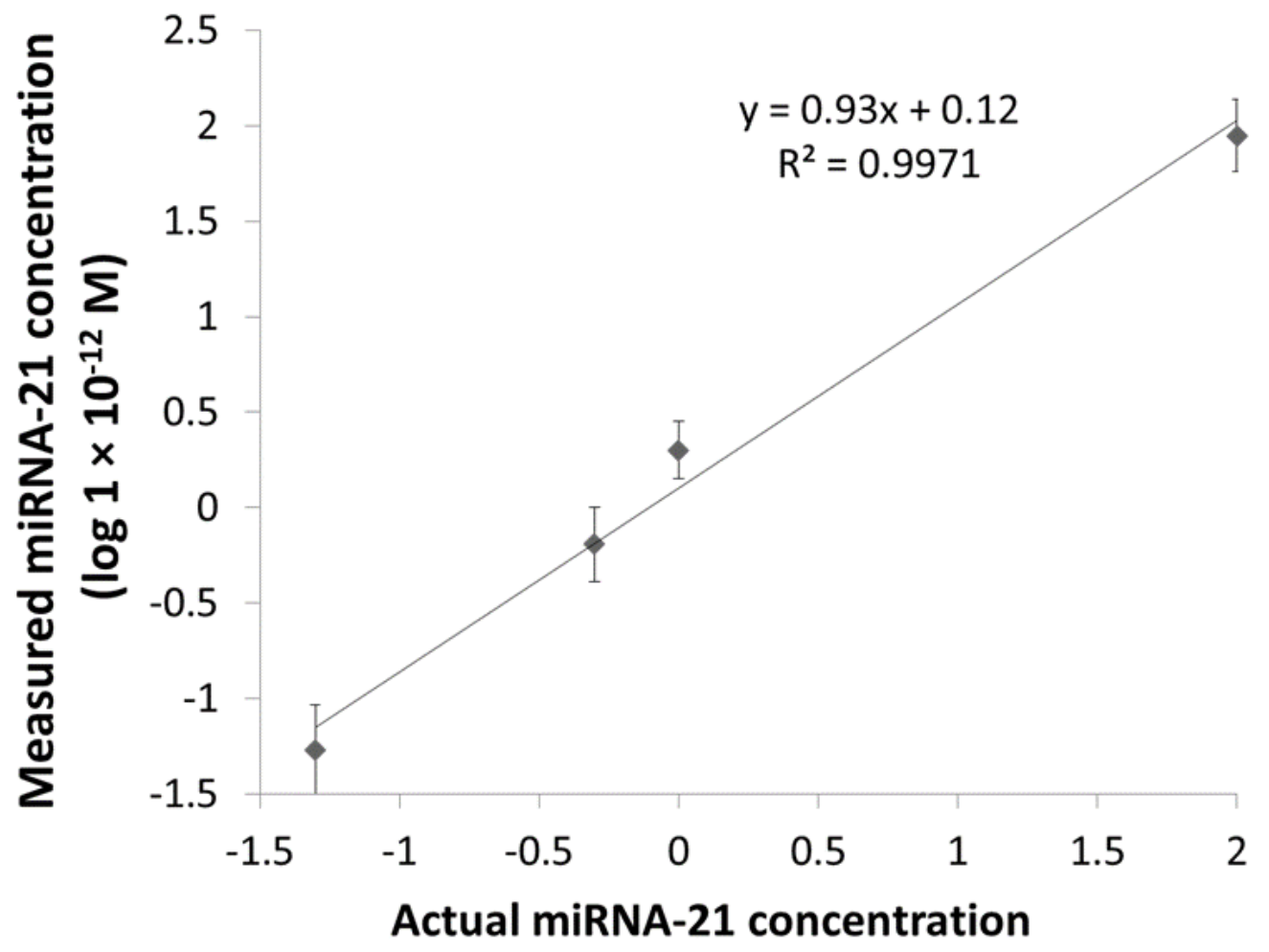

